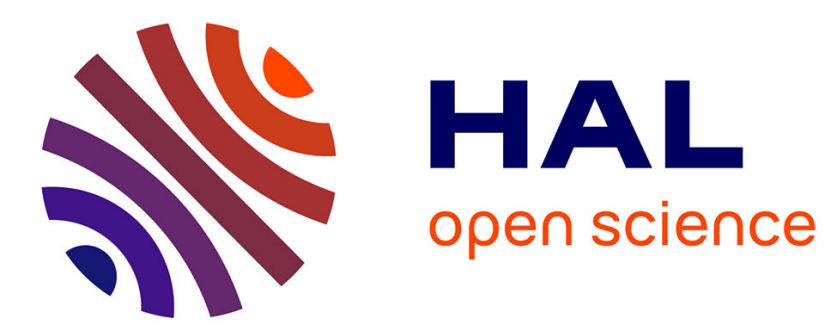

\title{
Experimental and numerical study of the turbulence characteristics of air flow around a research vessel
}

\author{
S. Popinet, M. Smith, C. Stevens
}

\section{To cite this version:}

S. Popinet, M. Smith, C. Stevens. Experimental and numerical study of the turbulence characteristics of air flow around a research vessel. Journal of Atmospheric and Oceanic Technology, 2004, 21 (10), pp.1574-1589. 10.1175/1520-0426(2004)0212.0.CO;2 . hal-01445435

\section{HAL Id: hal-01445435 \\ https://hal.science/hal-01445435}

Submitted on 24 Jan 2017

HAL is a multi-disciplinary open access archive for the deposit and dissemination of scientific research documents, whether they are published or not. The documents may come from teaching and research institutions in France or abroad, or from public or private research centers.
L'archive ouverte pluridisciplinaire HAL, est destinée au dépôt et à la diffusion de documents scientifiques de niveau recherche, publiés ou non, émanant des établissements d'enseignement et de recherche français ou étrangers, des laboratoires publics ou privés. 


\title{
Experimental and numerical study of the turbulence characteristics of air flow around a research vessel
}

\author{
Stéphane Popinet* Murray Smith ${ }^{\dagger}$ \\ Craig Stevens ${ }^{\ddagger}$ \\ National Institute of Water and Atmospheric Research, \\ PO Box 14-901 Kilbirnie, Wellington, New Zealand \\ Submitted to Journal of Atmospheric and Oceanic Technology
}

October 1, 2003

\begin{abstract}
Airflow distortion by research vessels has been shown to significantly affect micro-meteorological measurements. This study uses an efficient time-dependent Large Eddy Simulation numerical technique to investigate the effect of the research vessel Tangaroa on both the mean and turbulent characteristics of airflow. Detailed comparison is given between the numerical results and an extensive experimental dataset. The study is performed for the whole range of relative wind directions and for instruments located in regions of high and low flow distortion. The experimental data show that both the normalised wind speed and normalised standard deviation are only weakly dependent on wind speed, ship speed, ship motion and sea state, but strongly dependent on relative wind direction. Very good agreement is obtained between the experimental and numerical data for the mean flow, standard deviation and turbulence spectra, even in areas of strong turbulence.
\end{abstract}

\section{Introduction}

Interactions between the atmosphere and ocean form an important part of the climate system. Measurement of fluxes of momentum, heat, gases, and the accurate parameterisation of those fluxes, are required for global climate and weather models. Research vessels are the most common measuring platform for these flux measurements but they are also susceptible to airflow distortion effects around the hull and superstructure. It is therefore important to understand and accurately model the influence of the sampling platform on airflow and turbulence.

Yelland et al. (1998) examined two airflow effects: a) the acceleration/deceleration of flow and b) the tilt of the flow that results in air with turbulent characteristics from a different height being measured. These effects influence the drag coefficient for neutral atmospheric stability $\left(C_{D_{10 N}}\right)$ calculated using the inertial dissipation method. This method is preferred at sea since it uses frequencies in the inertial subrange of turbulence that are well above the frequencies associated with the wave-induced platform motion. The drag coefficient is given by:

$$
C_{D_{10 N}}=\frac{(k z \epsilon)^{2 / 3}}{U_{10 N}^{2}}
$$

\footnotetext{
*s.popinet@niwa.co.nz

†m.smith@niwa.co.nz

‡c.stevens@niwa.co.nz
} 
where $k$ is the von Karman constant, $z$ is the height at which the air originated, $\epsilon$ is the turbulent dissipation rate (determined from spectra of wind fluctuations), and $U_{10 N}$ is the wind speed at 10$\mathrm{m}$ height under neutral conditions. The bias in $C_{D}$ arises from $U_{10 N}^{2}$ but also more weakly from $z^{2 / 3}$ through uplifting of airflow. Yelland et al. (1998) showed that the resulting bias on the drag coefficient could be as large as $60 \%$. They also concluded that the azimuthal dependence of flow distortion could explain much of the open ocean variation of wind stress between experiments which had formerly been attributed to a wave-age effect.

Various attempts have been made to model the airflow distortion effects using both physical and numerical models. Thiebaux (1990) used wind tunnel tests to show that flow over Canadian research ships was accelerated by $7 \%$ at the position of the ship's anemometer above the bridge. Brut et al (2002) performed scale model simulations in a water flume at various static angles of pitch and azimuthal wind direction. In particular they showed that a heavily-instrumented mast can have strong effects.

However physical models have their limitations. While they can be used effectively to describe mean flow distortion, in order to model the distortion effects on turbulent fluxes, the turbulent length scale must also be scaled. This has proved difficult to achieve (Oost et al. 1994).

Potential flow estimates of flow distortion have been used by Kahma and Lepparanta (1981) and Oost et al (1994). However the first three-dimensional computational fluid dynamics (CFD) modelling was carried out by Yelland et al. (1998) for RSS Discovery and RSS Charles Darwin, using the commercially available package, Vectis. A single bow-on flow direction was examined. Yelland et al. (2002) extended this work with simulated flows at five different angles to the bow, from -30 to +30 degrees of the bow. Bow-on flows were also carried out for several other research ships. The main conclusion of their work was that model-derived corrections for mean flow distortion and vertical displacement of flow are essential for the calculation of $C_{D_{10 N}}$ to avoid biases greater than $20 \%$. The study considered mean flow properties only, not the turbulence properties.

Recently Dupuis et al (2003) presented CFD model results for L'Atalante using the Fluent $5 \mathrm{nu}$ merical model at a range of six azimuth angles from 0 to 180 degrees of the bow. As with Yelland et al. (2002) they found that the wind speed errors were independent of wind speed. Dupuis et al. also found no significant difference between using k-epsilon conditions and laminar flow, which allows a considerable saving in computation time.

While these studies have examined the distortion of the time-averaged flow, the effects on the turbulent structures are largely unknown (Oost et al. 1994). Questions remain about the distortion of turbulence by the flow disturbance, phase angles between $\mathrm{u}, \mathrm{w}$ (Oost et al. 1994; Pedreros et al. 2003), generation of turbulent vortices by superstructures and anemometer support structures.

Our aims here are:

- develop a robust, efficient CFD model which is able to give access to the time-dependent turbulent characteristics of the flow,

- evaluate the performance of this model for mean airflow distortion effects against shipborne measurements with an emphasis on areas of high flow distortion (turbulent wakes and recirculation zones),

- examine initial CFD results of time-dependent turbulence generated by flow interaction with the ship superstructure.

In Section 2 we describe the experimental monitoring during the experimental voyage. Section 3 gives an overview of the main features of the Large-Eddy Simulation (LES) numerical technique we developed. Section 4 presents a summary and analysis of the experimental data and section 5 includes a detailed comparison of the experimental data with numerical simulations spanning the whole range of relative wind directions (from bow-on to stern-on) for both mean and turbulent flow properties. 


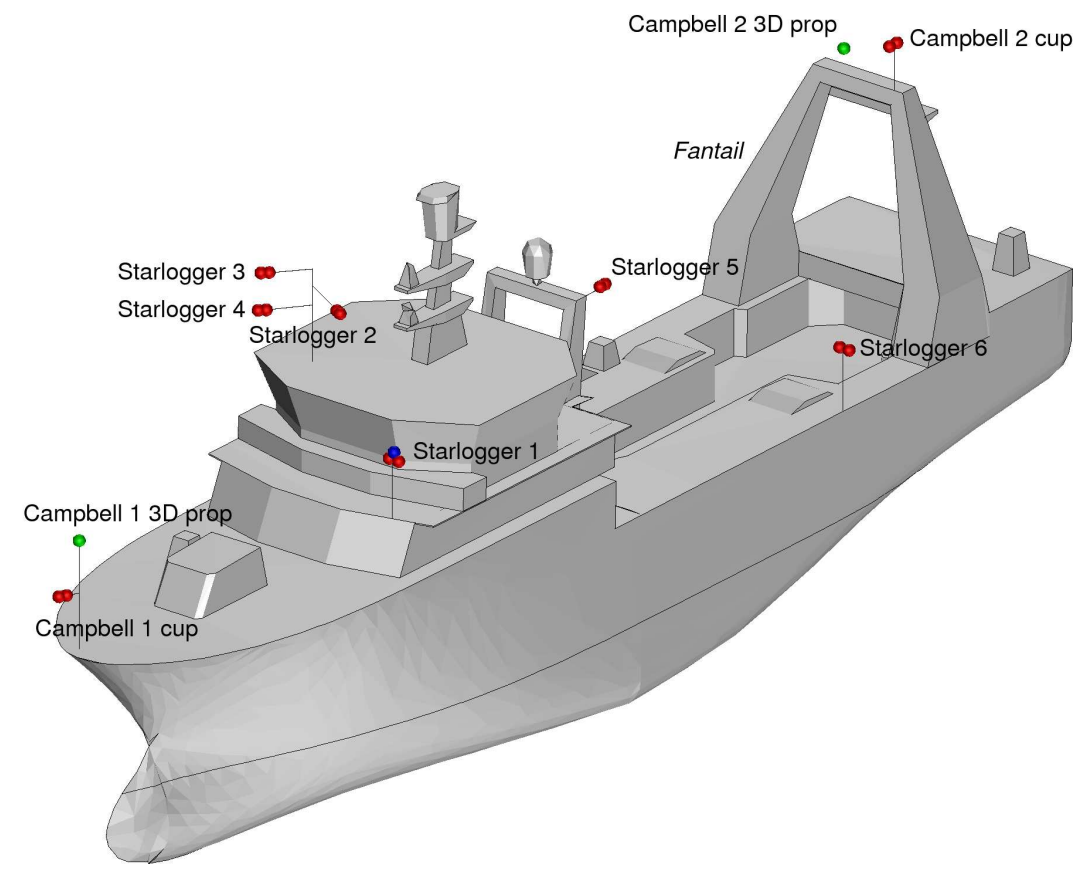

Figure 1: A CAD representation of the Tangaroa used in the present simulations. Location of the validation instruments are also marked.

\section{Experimental setup}

Eight Vector cup/vane anemometers and two robust Weathertronics 3D propeller anemometers were installed on RV Tangaroa. Figures 1, 2 and table 1 illustrate the location of the instruments. Several instruments (Campbell 1 3D prop, Campbell 2 3D prop, Starlogger 3) are located in sites which could possibly be used as permanent sampling sites. The other instruments are deliberately located in areas of flow which are likely to be strongly affected by the ship (in front and behind the central superstructure).

For practical reasons, several instruments are also mounted on short booms and thus lie relatively close to the ship. In particular, we expect Starlogger 2, 3 and 4 to sample the strong velocity gradients caused by flow separation above the central superstructure. Together with instruments located in the turbulent wake of the central superstructure (Starlogger 5 and 6) this will provide a stringent test of the numerical method. All the cup/vane anemometers were sampled every three seconds, while the

\begin{tabular}{rr|cr} 
Instrument & Elevation (m) & Instrument & Elevation (m) \\
\hline $\begin{array}{r}\text { Starlogger 1 } \\
\text { cup/vane }\end{array}$ & 13.8 & $\begin{array}{c}\text { Starlogger 6 } \\
\text { cup/vane } \\
\text { Campbell 1 } \\
\text { cup/vane }\end{array}$ & 8.7 \\
$\begin{array}{r}\text { Starlogger 2 } \\
\text { cup/vane }\end{array}$ & 18.4 & $\begin{array}{c}\text { 3D prop } \\
\text { Campbell 2 }\end{array}$ & 11.5 \\
$\begin{array}{r}\text { Starlogger 3 } \\
\text { cup/vane }\end{array}$ & 19.4 & $\begin{array}{c}\text { cup/vane } \\
\text { Starlogger 4 }\end{array}$ & \\
cup/vane & 17.4 & 3D prop & 19.6 \\
Starlogger 5 & & & 19.9 \\
cup/vane & 15.6 & &
\end{tabular}

Table 1: Elevation above sea level of the instruments. 


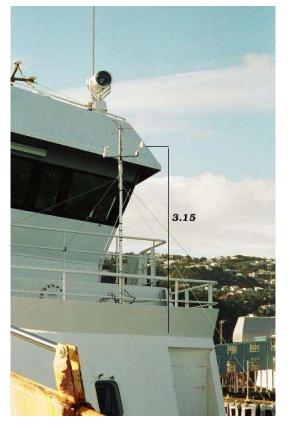

Starlogger 1

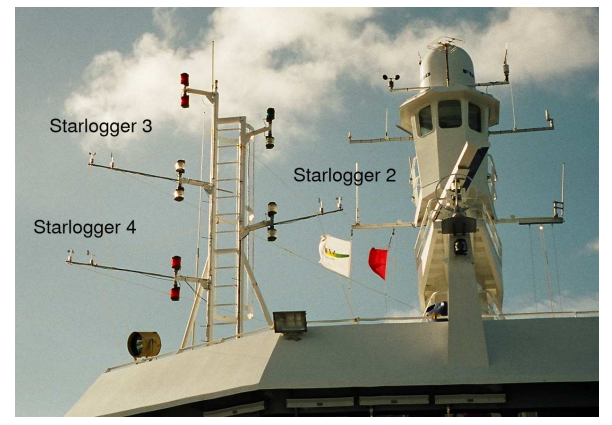

Starlogger 2, 3, 4

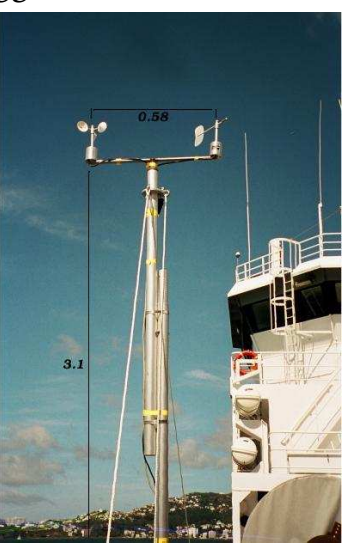

Starlogger 6

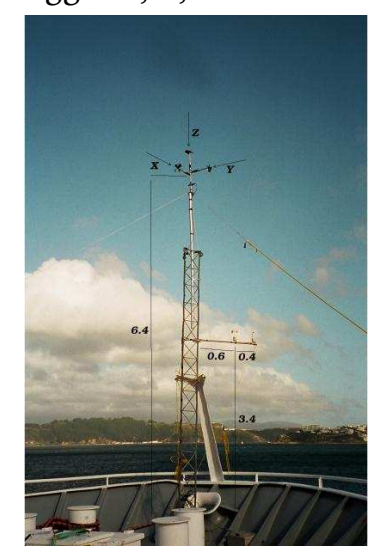

Campbell 1 3D prop, cup

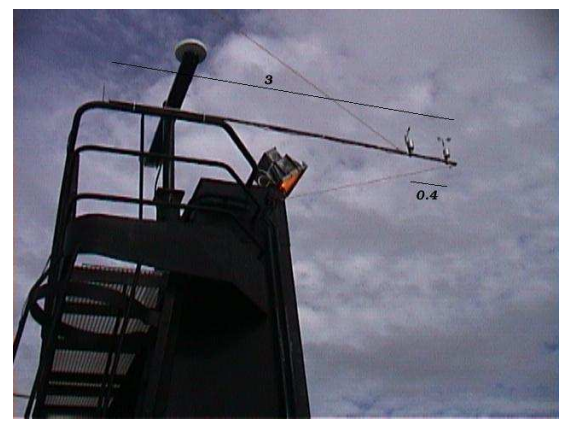

Starlogger 5

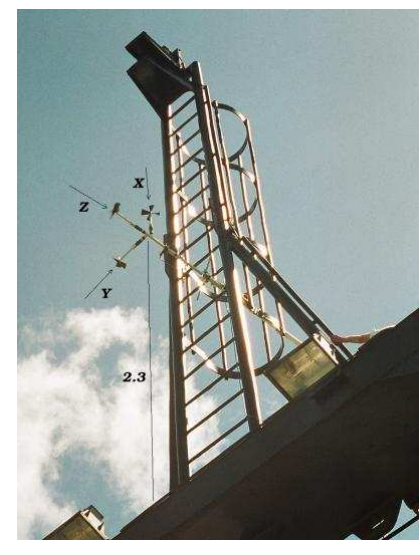

Campbell 2 3D prop

Figure 2: Individual mountings of instruments marked in figure 1. 


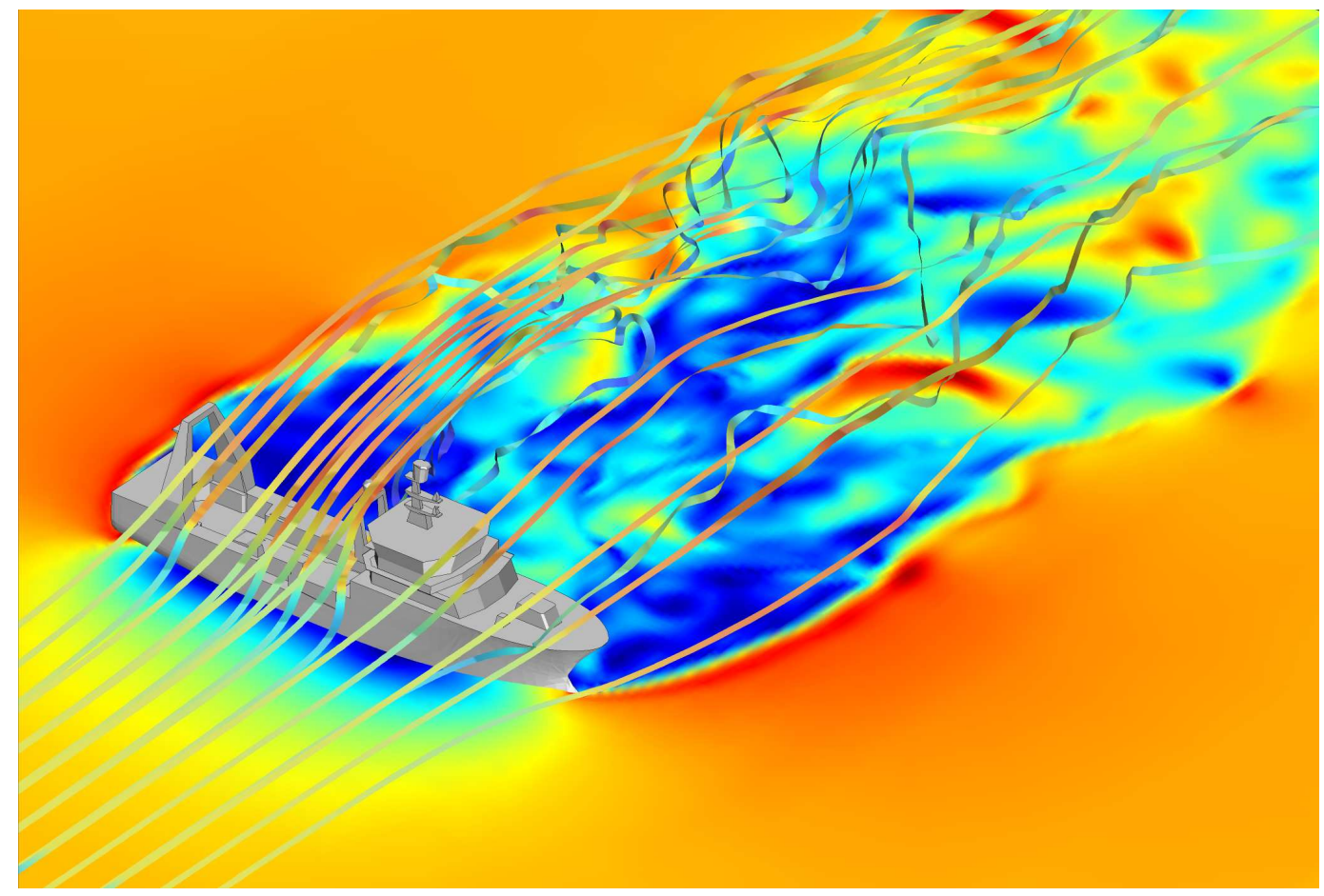

Figure 3: Side-on wind flow. The stream ribbons and cross-section at sea level are coloured according to the norm of the velocity.

3D propellers were sampled at four Hertz.

\section{Numerical method}

Most CFD programs used for engineering applications provide a solution of the Reynolds averaged Navier-Stokes equations (RANS) where the averaging is carried out in space and time. The solution obtained is thus a stationary, time-averaged representation of the flow and provides only limited information on the turbulence characteristics. Another possibility is to carry out the averaging only spatially. The resulting time-dependent solution is then obtained using methods usually referred to as Large Eddy Simulations (LES). While these methods can be more computationally expensive, they require fewer assumptions for modelling turbulent stresses and have the potential to provide better solutions, particularly in wakes or recirculating regions (Shah and Ferziger 1997; Rodi et al. 1997) or around the bluff bodies we are interested in (Baetke et al. 1990; Murakami 1993).

The numerical method we used has been described in detail in Popinet (2003). Its implementation is freely available (Popinet 2002). In the following we summarise the main characteristics of the technique.

\section{a. Spatial discretisation}

The computational domain is discretised using cubic finite volumes organised as a spatial octree (Samet 1989; Khokhlov 1998). This type of discretisation is very flexible and allows the spatial resolution to dynamically adapt to follow the evolving flow structures (Popinet 2003). An example of such a discretisation is given in figure 4 . The wake created by the ship for a side-on wind flow is resolved using the finest mesh. Far from the ship, only large structures are present and the spatial resolution decreases accordingly. The mesh is adapted at each time step to follow the evolving turbulent boundary of the wake.

This mechanism allows substantial savings in computation time. Fine meshes can thus be used to 


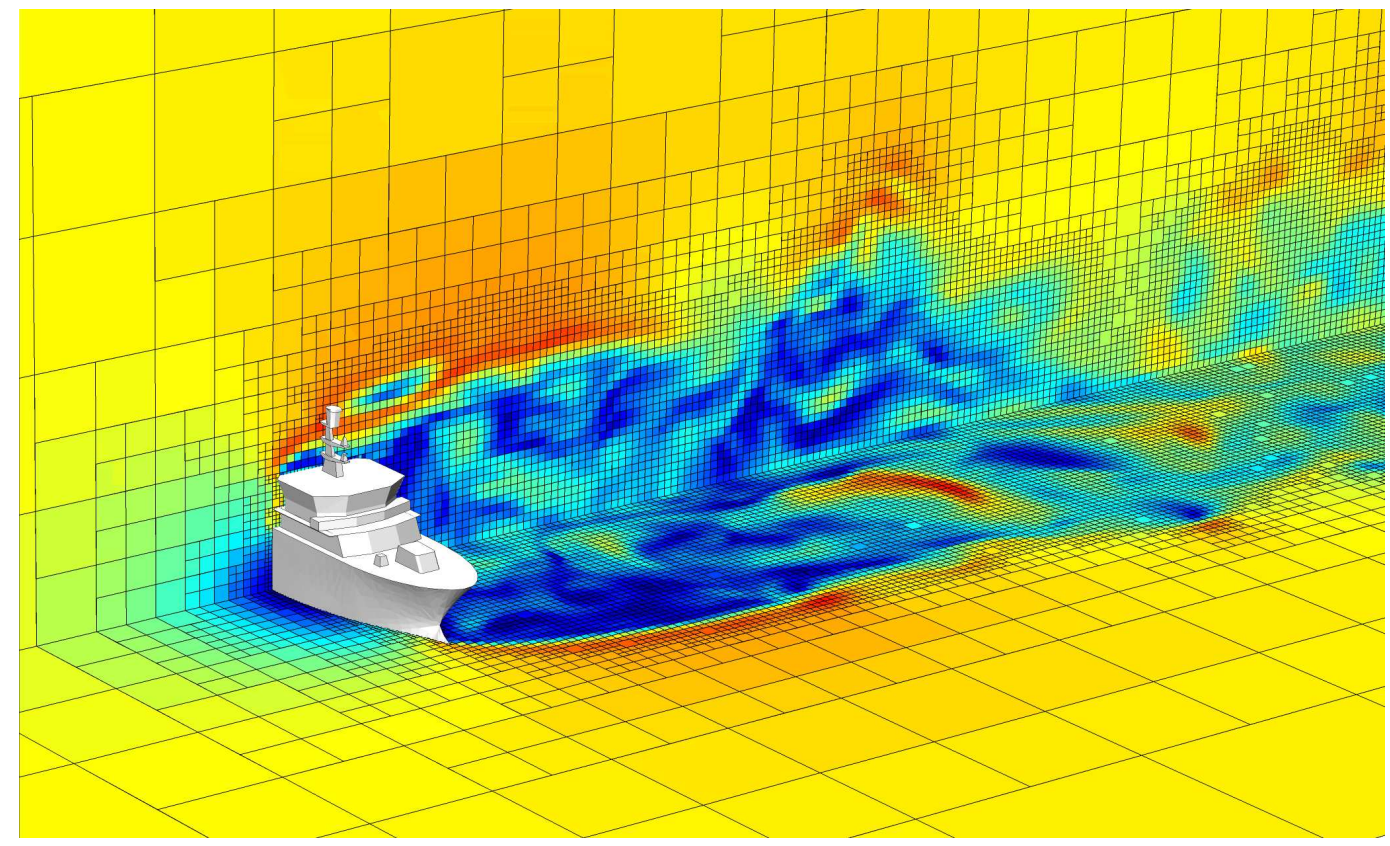

Figure 4: Adaptive mesh used for the side-on wind flow. The horizontal and vertical cross-sections illustrate the three-dimensional adaptive octree.

resolve the ship geometry and the small turbulent structures it creates. A drawback is that, contrary to traditional unstructured finite-element/finite-volume techniques, the boundary of the discretised volumes cannot be made to correspond with complex boundaries. This problem can be solved by using "cut-cell" techniques (Quirk 1994; Almgren et al. 1997; Ye et al. 1999) which take into account the exact shape of finite-volumes cut by the solid boundary. When implemented properly, these techniques have the added advantage of allowing simple automatic mesh generation, independently of the complexity of the solid boundaries considered.

\section{b. Temporal discretisation}

We consider a constant density, incompressible and inviscid fluid. Given a velocity field

$$
\mathbf{U}(x, y, z, t)=(u(x, y, z, t), v(x, y, z, t), w(x, y, z, t)),
$$

and a pressure field $p=p(x, y, z, t)$ defined at location $(x, y, z)$ and time $t$, on some domain $\Omega$ with a solid wall boundary $\partial \Omega$, the incompressible Euler evolution equations for $\mathbf{U}$ are

$$
\begin{aligned}
\mathbf{U}_{t} & =-u \mathbf{U}_{x}-v \mathbf{U}_{y}-w \mathbf{U}_{z}-\nabla p, \\
\nabla \cdot \mathbf{U} & =0 .
\end{aligned}
$$

The boundary condition for the velocity at solid wall boundaries is the no-flow condition

$$
\mathbf{U}(x, y, z, t) \cdot \mathbf{n}=0 \text { for }(x, y, z) \in \partial \Omega,
$$

where $\mathbf{n}$ is the outward unit vector on $\partial \Omega$.

We use a classical fractional-step projection method (Chorin 1968; Peyret and Taylor 1983; Brown et al. 2001). At any given timestep $n$, we assume that the velocity at time $n$, $\mathbf{U}^{n}$ and the fractional step pressure $p^{n-1 / 2}$ are known at cell centres. In a first step, a provisional value $\mathbf{U}^{\star \star}$ is computed using

$$
\frac{\mathbf{U}^{\star \star}-\mathbf{U}^{n}}{\Delta t}=-\mathbf{A}^{n+1 / 2}
$$


where $\mathbf{A}^{n+1 / 2}$ is an approximation to the advection term $[(\mathbf{U} \cdot \nabla) \mathbf{U}]^{n+1 / 2}$. The new velocity $\mathbf{U}^{n+1}$ is then computed by applying an approximate projection operator to $\mathbf{U}^{\star \star}$ which also yields the fractional step pressure $p^{n+1 / 2}$ (Almgren et al. 2000).

The advection term $\mathbf{A}^{n+1 / 2}$ is computed using a second-order, unconditionally stable, Godunovtype scheme (Bell et al. 1989), with a cell-merging technique for small cut cells (Quirk 1994). The overall scheme is thus second-order in space and time.

\section{c. Poisson equation}

The projection method relies on the Hodge decomposition of the velocity field as

$$
\mathbf{U}^{\star \star}=\mathbf{U}+\nabla \phi
$$

where

$$
\nabla \cdot \mathbf{U}=0 \text { in } \Omega \text { and } \mathbf{U} \cdot \mathbf{n}=0 \text { on } \partial \Omega .
$$

Taking the divergence of (2) yields the Poisson equation

$$
\nabla^{2} \phi=\nabla \cdot \mathbf{U}^{\star \star}
$$

while the normal component of (3) yields the boundary condition

$$
\frac{\partial \phi}{\partial \mathbf{n}}=\mathbf{U}^{\star \star} \cdot \mathbf{n} \text { on } \partial \Omega .
$$

The divergence-free velocity field is then defined as

$$
\mathbf{U}=\mathbf{U}^{\star \star}-\nabla \phi
$$

where $\phi$ is obtained as the solution of the Poisson problem (4). This defines the projection of the velocity $\mathbf{U}^{\star \star}$ onto the space of divergence-free velocity fields.

This projection step is the most expensive part of the solution algorithm because equation (4) results in a spatially implicit problem (i.e. a linear system of equations for each discrete volume). We use an efficient multigrid-accelerated relaxation technique which combines naturally with the octree spatial discretisation (Popinet 2003).

\section{d. Turbulence modelling}

Given the very high Reynolds number of a typical airflow around a ship $\left(\mathcal{R} \approx 10^{9}\right)$ direct numerical simulations are not feasible: the scale of the smallest possible structures (the Kolmogorov scale) being of the order of $1 / \mathcal{R}$. Some turbulence modelling is thus necessary to approximate the energy transfer at scales smaller than the mesh size. In Large Eddy Simulations (LES) this subgrid energy transfer is usually assumed to take the form of a subgrid turbulent viscous stress where the viscosity coefficient is variable both in space and time and described using semi-empirical relationships (Lesieur and Métais 1996).

As described above, the numerical model we use does not contain any explicit viscous terms. In practice numerical schemes always have some numerical viscosity due to higher order errors associated with the discrete representation of the solution. Remarkably, several authors (Boris et al. 1992; Porter et al. 1994) have shown that this numerical dissipation can describe turbulent subgrid energy transfer as well or sometimes better than more complex LES semi-empirical models. Consequently, this first study will not use any explicit turbulent dissipation, while we certainly do intend to investigate more complex LES models in the future.

\section{Experimental Results}

Data was collected continuously during a week long cruise in the Pacific ocean, south-east of the New Zealand mainland, during March 2002 (see figure 5). A range of wind speeds, up to $20 \mathrm{~m} / \mathrm{s}$, was sampled with sea conditions and ship motion varying accordingly (from calm seas to up to 


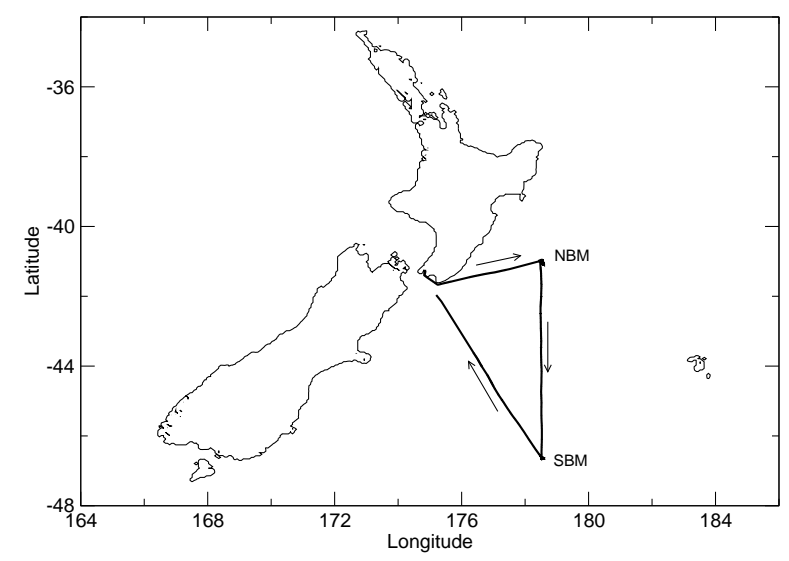

Figure 5: Track of the ship position.

6-7 meters swells). Figure 6.(a) illustrates the variability of the measured relative wind speed at location "Campbell $13 \mathrm{D}$ prop" during the whole window of observation we consider in this study. The corresponding ship speed is given in figure 6.(b). The data sampled are roughly evenly distributed between periods where the ship was stationary (while carrying out maintenance work on moorings at locations NBM and SBM) and periods with a cruising speed of around $6 \mathrm{~m} / \mathrm{s}$.

In order to obtain a synthetic representation of all the data collected, we made two assumptions:

1. the wind speeds measured at the different locations should scale linearly with some reference velocity i.e. the fluid flow is essentially independent from the Reynolds number.

2. this normalised velocity depends only on the relative wind direction.

The first assumption is justified as the Reynolds number is very high $\left(\approx 10^{9}\right)$ well within the asymptotic regime for flow around a solid obstacle. The second assumption is much stronger in that we have chosen to neglect the influence of sea conditions as well as ship motion. More specifically, we chose not to take into account the difference in vertical (apparent) wind profiles as seen from a moving or stationary ship.

To apply these assumptions to the data, we need to choose a reference velocity. Ideally one would have access to some reference measurement away from the ship. As we do not have such reference, we chose to use both the "Campbell 1" 3D propeller data and the "Campbell 2" cup anemometer data. Being farthest away from the ship superstructure, these instruments are the best possible approximation to a reference measurement. This is of course not the case when either of them lie directly in the wake of the central superstructure. We avoid this problem by taking the Campbell 1 site as reference for all relative wind directions in $[-110,+110]$ degrees and Campbell 2 otherwise. The relative wind direction is defined in the same way.

Figure 7 illustrates the dependence of the relative wind speed measured at each location on the relative wind direction. Each dot is a one minute average of the experimental time series. All the data for the time window considered (from the 17th to the 22nd of March 2002) is represented (around 6000 samples for each graph). A very clear relationship is obtained for all the measurement locations, the standard deviations being of the order of $5 \%$ of the reference velocity. This confirms that the assumptions we made are justified despite the wide range of sea conditions, ship and wind speeds. Regarding the structure of the variations observed, a first general observation is that we expect the relationships to be symmetrical around 180 degrees for instruments close to the centreline of the ship (the ship being roughly right/left symmetrical). This is indeed the case for the Starlogger 2 and Campbell 1 cup instruments, while others show varying degrees of asymmetry.

A number of distinctive features can be seen which are easy to relate to the geometry of the ship. The strong variations in velocities near 90 and 270 degrees for Starlogger 1, 2, 3, 4 and 5 can be linked directly to the flow upstream of the instruments moving behind local obstructions (mainly from the centre deck). Similarly the strong decrease in velocity near 180 degrees for Campbell 1 cup 


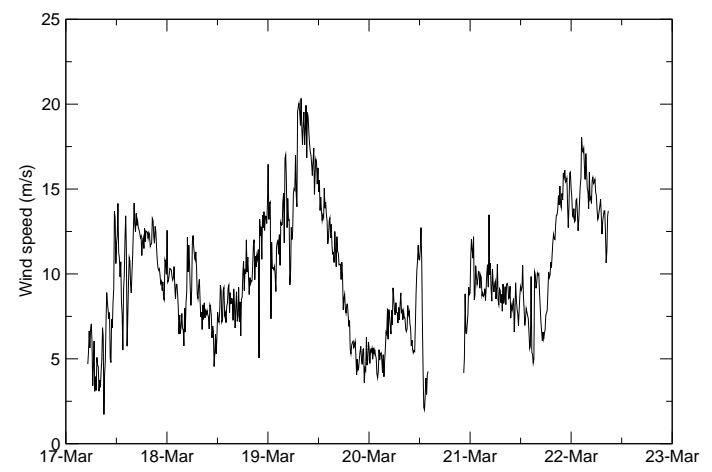

(a)

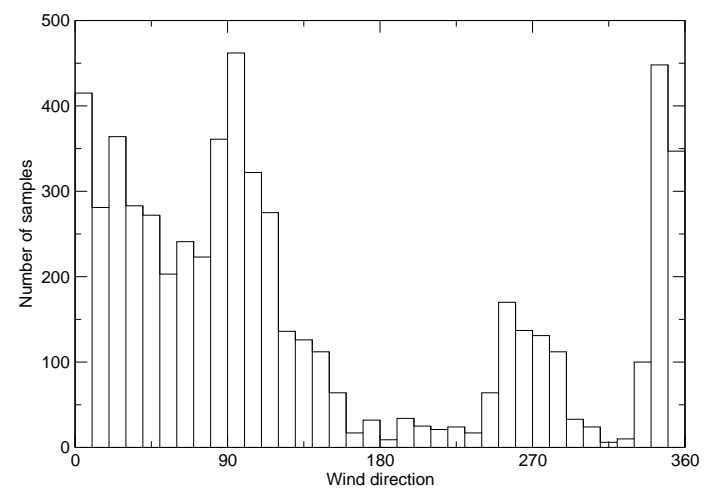

(c)

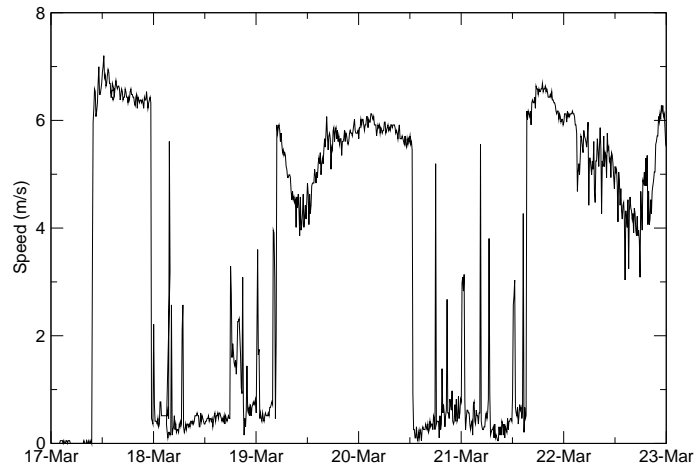

(b)

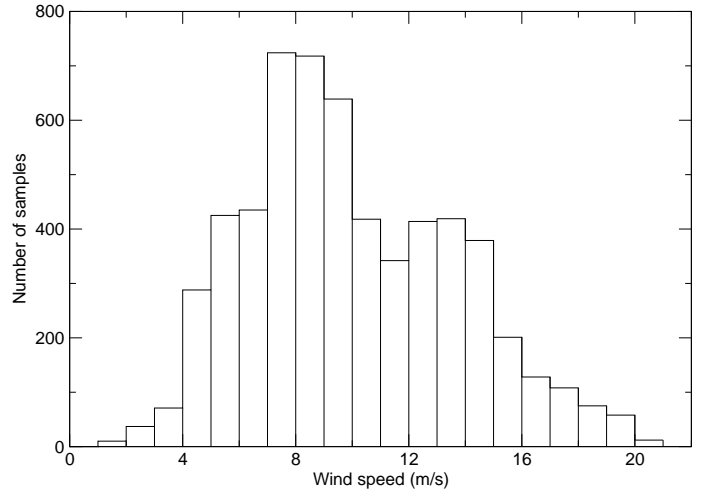

(d)

Figure 6: (a) Measured relative wind speed at the "Campbell 1 3D prop" location. (b) Ship speed. (c) Distribution of relative wind directions. (d) Distribution of wind speeds. 

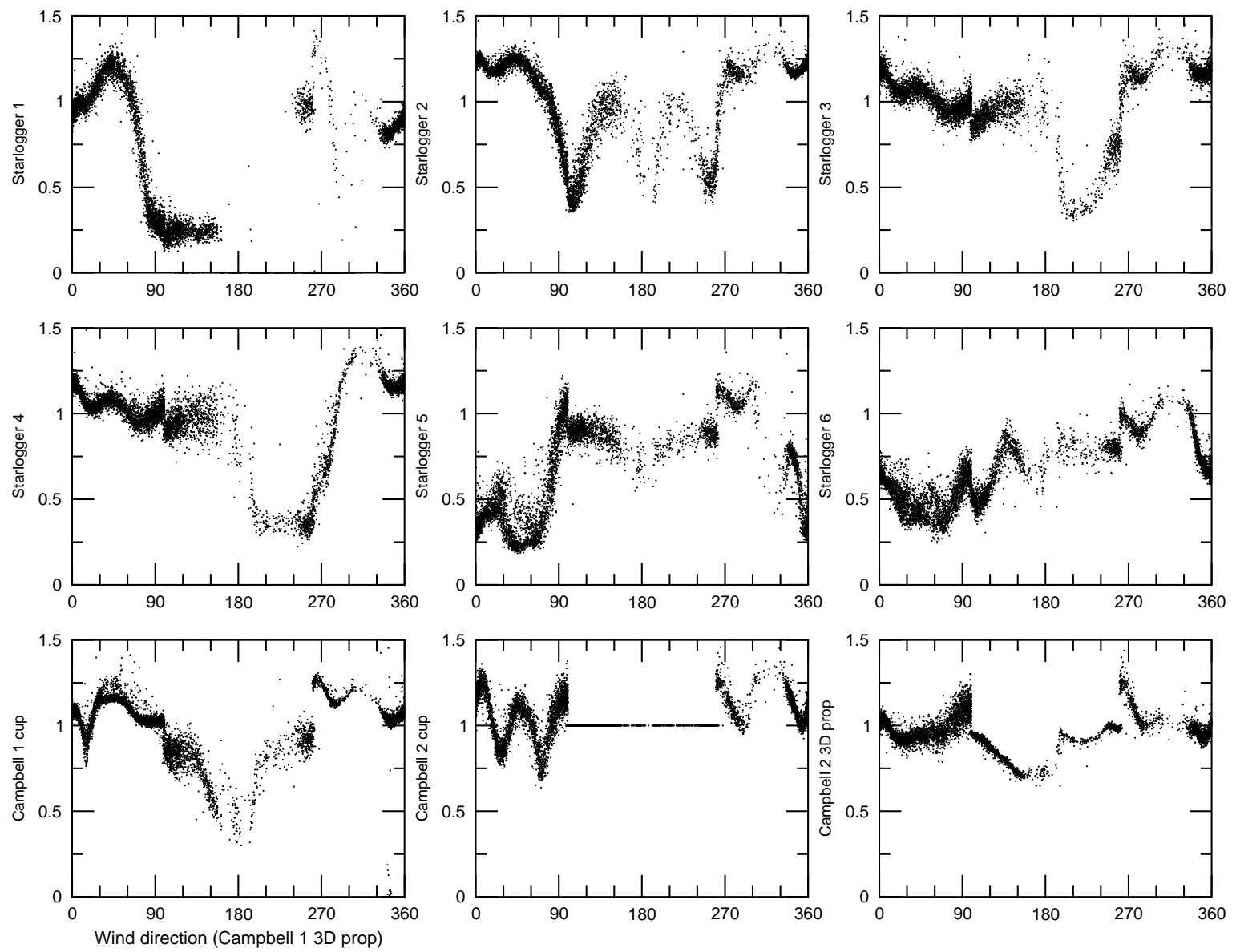

Figure 7: Normalised relative wind speeds measured at each location as functions of relative wind direction (reference Campbell 1 3D prop / Campbell 2 Cup). Each dot is a one minute average. 
can be associated with the instrument being in the wake of the central superstructure. The structure of the dependence for most of the instruments can be characterised by two regimes:

- a more or less "laminar" regime where the instrument sits in a relatively undisturbed flow (possibly with some potential flow acceleration) e.g. Starlogger 1 and 2 between 0 and 75 degrees, Starlogger 3 and 4 between 0 and 180 degrees.

- a strongly turbulent regime where the instruments sits downstream of an obstruction e.g. Starlogger 1 and 2 above 90 degrees, Starlogger 3 and 4 between 180 and 270 degrees.

Starlogger 6 is a particular case where only the turbulent regime is present, the instrument almost always being downstream of some obstruction.

Some features of Campbell 1 cup deserve particular attention. This instrument is located immediately below Campbell 1 3D prop, used as reference for all "bow-on" winds. One would thus expect a nearly constant relationship for all bow-on angles, but a sharp decrease is observed around 15 degrees as well as other well-defined structures near 270 degrees. Apart from systematic instrumental errors (improbable given the well-defined features and wide range of wind speeds), a probable explanation is the influence on the measured wind speed of small-scale details like mast mounting and fittings.

\section{Comparison with numerical simulations}

\section{a. Numerical setup}

Using the numerical method previously described we performed a number of simulations of the flow around a CAD model of RV Tangaroa for different relative wind directions. The CAD model used is pictured in figure 1. We aimed for a spatial resolution near the ship of around 50 centimetres. Consistently the smallest details represented in the CAD model are of this order. In order to minimise the influence of the boundary conditions, the CAD model is positioned in a cubic domain 276 metres wide (four times the ship length). The corresponding maximum blockage ratio obtained for beam-on flows is of the order of one percent. A constant, unity inflow velocity is imposed to the left side of the domain, simple outflow conditions to the right side and slip conditions on all the other boundaries (including the sea surface). As in Dupuis et al (2003), we chose not to impose a more complex velocity profile (logarithmic boundary layer or other models) at the inflow for two reasons:

1. we solve the Euler equations and thus cannot impose the explicit dissipative terms consistent with a non-zero stress at the sea surface.

2. the experimental results have shown that the flow distortion is largely independent of the ship motion and thus of the detail of the vertical wind velocity profile.

The simulations are all started with the potential flow solution as initial conditions. As time passes, the vorticity generated at the solid boundaries (essentially near sharp features of the CAD model) is advected away from the ship and evolves into a fully developed turbulent wake. The computational mesh is adapted dynamically to follow this evolution using the vorticity criterion (figure 4). We chose to refine the mesh in areas of high vorticity down to a spatial scale of one metre $(50 \mathrm{~cm}$ if close to the ship). Depending on the relative wind direction, between 200,000 and 350,000 grid points were necessary to resolve the fully developed turbulent wakes.

Typical results are illustrated in figure 8 and 9 for bow-on and stern-on flows respectively. The pictures are a snapshot in time of the fully-developed wake. The stream ribbons (streamlines twisted according to the local vorticity vector) pictured go through individual instrument locations. In figure 8 two regimes can be clearly distinguished: a laminar flow upstream of the central superstructure and a strongly turbulent flow downstream. The signature of this turbulent wake is clearly seen in the large fluctuation in wind velocity near sea level (coloured plane) while the close to potential flow solution upstream creates the characteristic low wind zone just upstream of the bow.

Since our model is time-dependent, it was necessary to select a window over which to timeaverage the numerical fields in order to compare the numerical results to the time-averaged experimental data of figure 7. We chose to stop the simulations at $t^{\star}=t U / L=3$ where $U$ is the inflow 


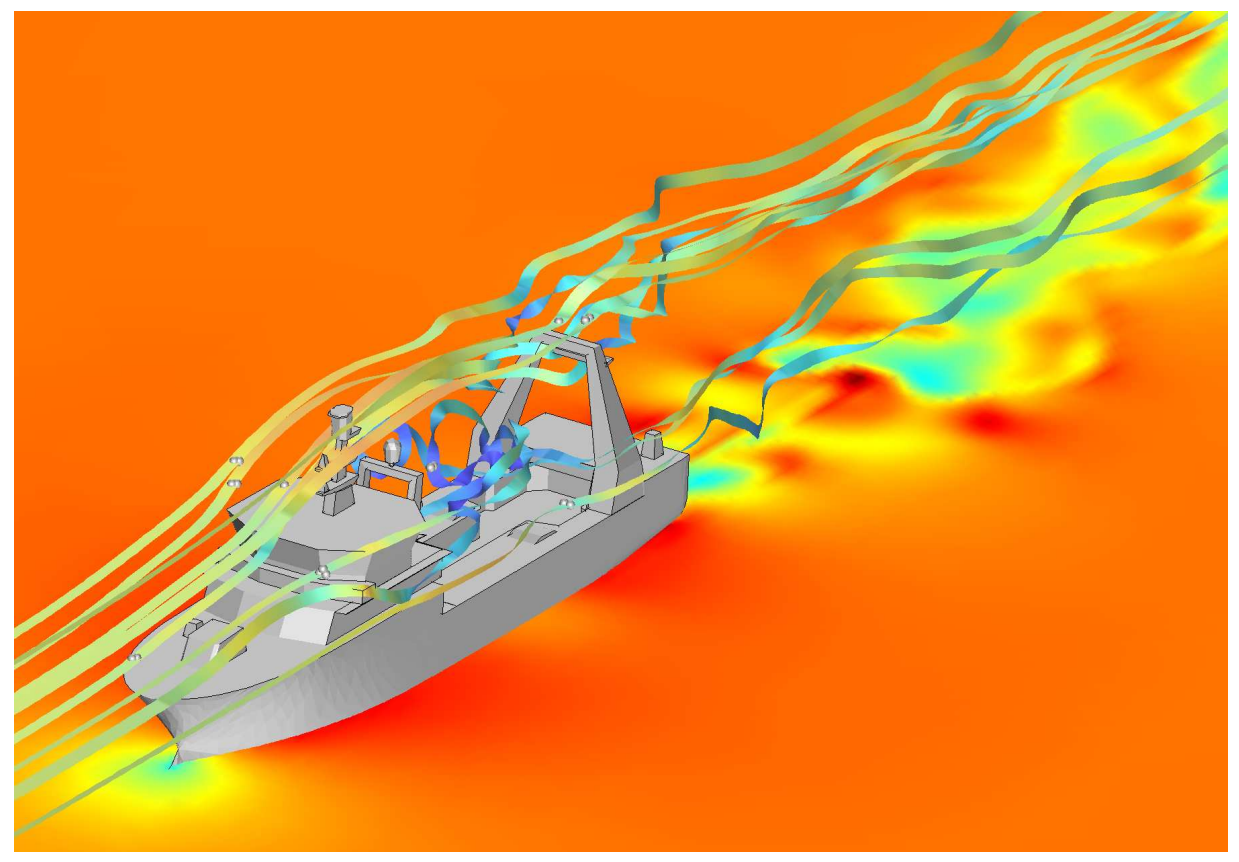

Figure 8: Streamlines passing through instruments locations for a bow-on wind. The sea surface and the streamlines are coloured according to the norm of the local wind velocity.

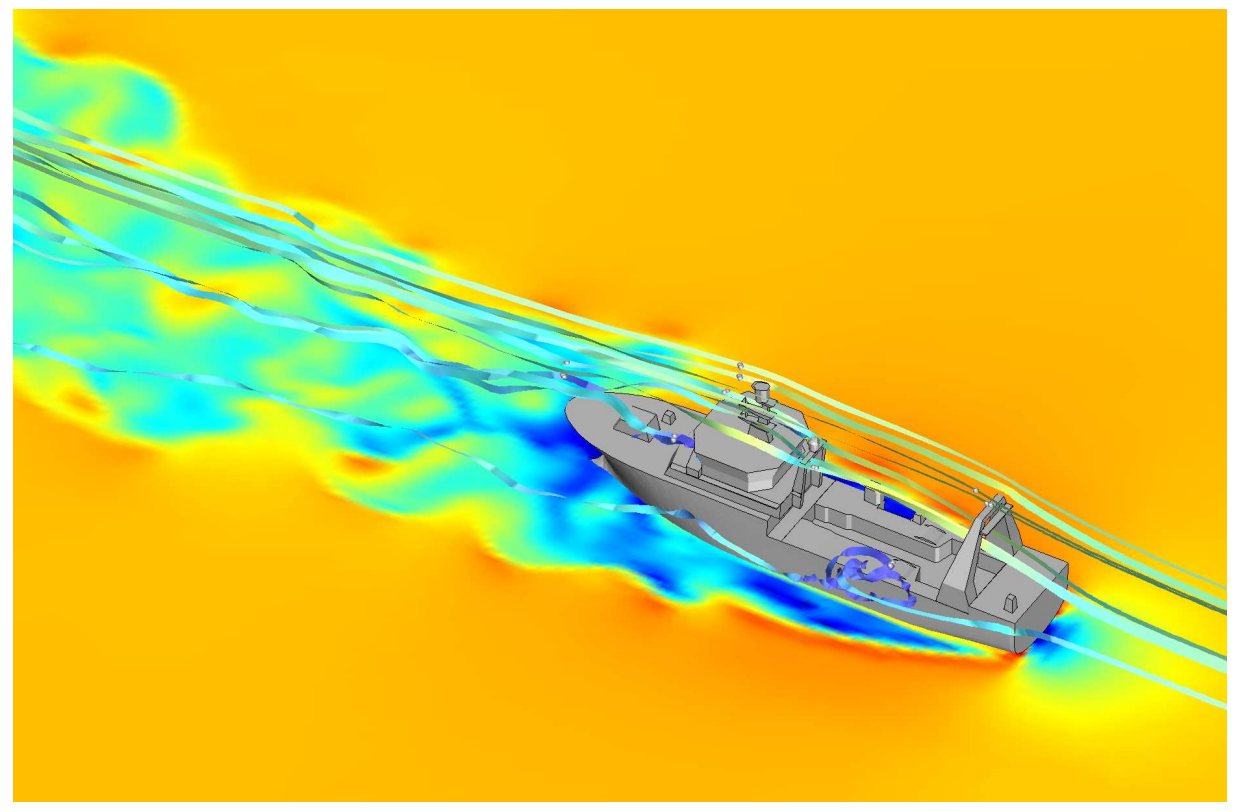

Figure 9: Streamlines passing through instruments locations for a stern-on wind. The sea surface and the streamlines are coloured according to the norm of the local wind velocity. Note the much wider wake compared to figure 8 . 

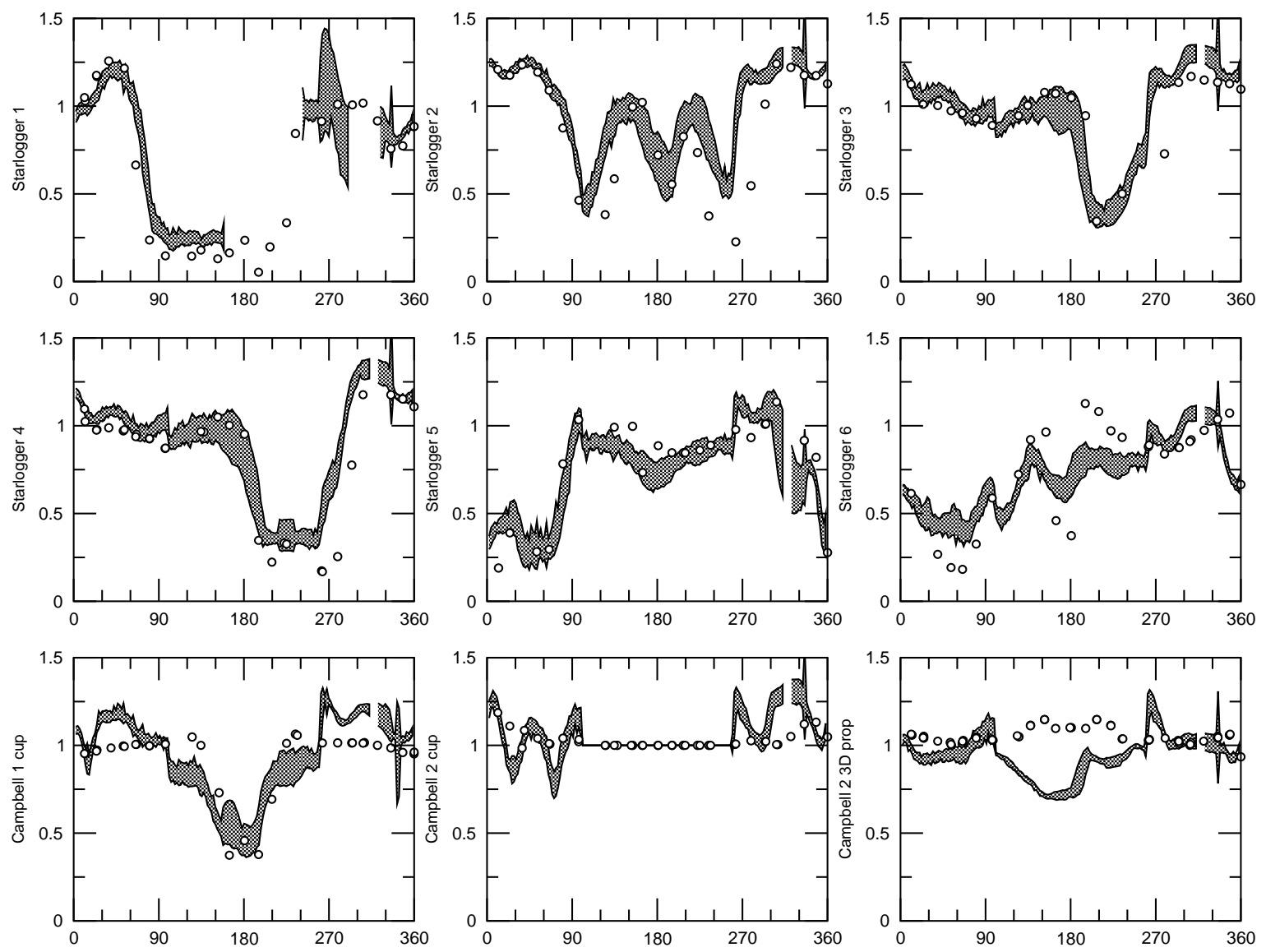

Figure 10: Relative wind speeds at each location as functions of relative wind direction. The experimental data is represented by the bounding curves defined by: mean plus or minus standard deviation. The symbols are the results of numerical simulations.

velocity and $L=276$ metres is the domain size, and to time-average the fields for $t^{\star} \in[1,3]$. One $t^{\star}$ unit was enough in all cases to obtain a fully-developed turbulent regime from the initial potential solution.

\section{b. Mean flow distortion}

A series of simulations were performed with a relative wind direction varying from 0 to 360 degrees by increments of 15 degrees. Each simulation took approximately 20 hours of CPU time on a $2 \mathrm{GHz}$ compatible PC. The results for the time-averaged relative wind speeds calculated at each instrument location are pictured in figure 10 together with the experimental data. For clarity, the experimental data of figure 7 is summarised here by the two curves: mean plus or minus standard deviation.

When looking more closely at the results, it is useful to distinguish the laminar and turbulent regimes. Very good agreement between the simulations and the experimental data is obtained in the laminar regime: 0 to 90 degrees for Starlogger 1 and 2 and 0 to 180 degrees for Starlogger 3 and 4. In the turbulent regime, good agreement is still obtained: correct low values for Starlogger 1 between 90 and 270 degrees, "M structure" for Starlogger 2, sharp gradients for Starlogger 3, 4 and 5. As noted earlier, Starlogger 6 is a difficult case, being located on the lower deck and always in a turbulent regime. While the general trend is reproduced by the model a number of small structures do not seem to match very well. A possible explanation is that several small scale structures (winches, railings, deck crane etc...) close to the instrument location are not represented in the CAD model. Similarly, the small structures in Campbell 1 cup and Campbell 2 cup which we attributed to local perturbations 


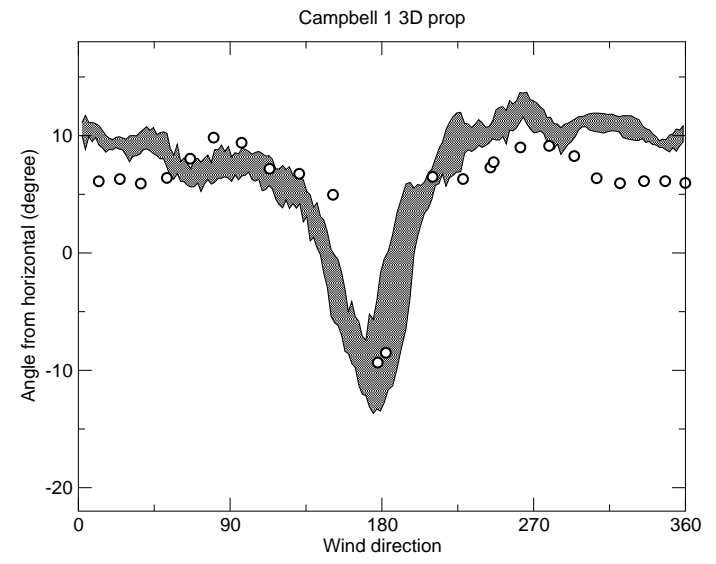

(a)

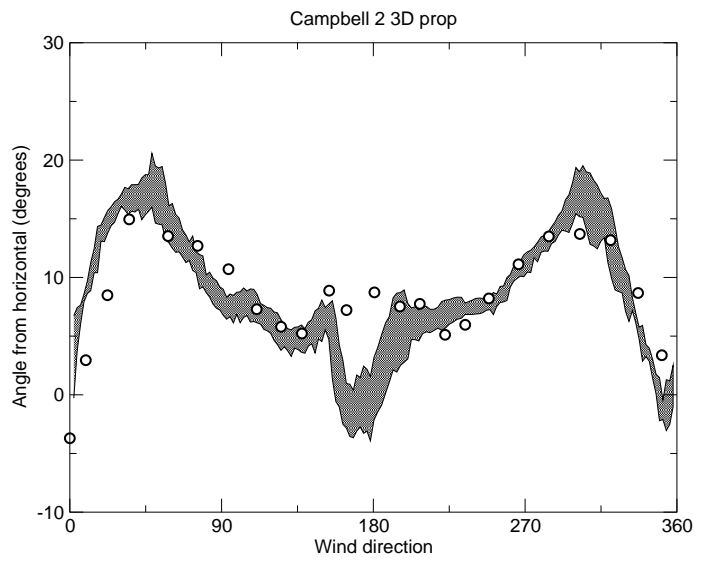

(b)

Figure 11: Angle from horizontal of the time-averaged velocity vector. The experimental data are represented by the bounding curves defined by: mean plus or minus standard deviation. The symbols are the results of numerical simulations. (a) "Campbell 1 3D prop" site. (b) "Campbell 2 3D prop" site.

are not reproduced by the numerical model. Overall and taking into account the variability of the experimental data, the agreement is very satisfying.

The two 3D propellers at locations Campbell 1 and Campbell 2 give an experimental measurement of the deviation of the flow from the horizontal. Figures 11.(a) and 11.(b) summarise the experimental and numerical results obtained at these two locations. Again, a well-defined experimental relationship is obtained. The numerical results match well with the experimental data. Strong gradients in angular deviation are well captured near 180 degrees for Campbell 1 and near 0 degrees for Campbell 2, while the total range of variation $([-10: 10]$ for Campbell 1 and $[-5: 15]$ for Campbell 2 ) is well reproduced. The systematic shifts of $\approx+5$ degrees for both locations can be attributed to an approximate alignment of the instruments (as can the slight asymmetry in relative wind direction for the measurements at Campbell 2). One feature which does not seem to be captured by the model is the decrease in angular deviation near 180 degrees at the Campbell 2 site (figure 11.(b)). For this relative wind direction, the 3D propeller is immediately downstream of the ladder (on top of the fantail) used as support, which may explain the discrepancy.

\section{c. Turbulence intensity}

The numerical simulations also provide detailed information about the structure of the turbulent wake. The turbulence intensity can be characterised by the standard deviation of the velocity measured at each location. Figure 12 gives a summary of the normalised standard deviation (using the same reference velocities as in figure 10) measured and simulated at each site. The laminar and turbulent regimes described for figure 10 are clearly apparent in this graph, with low average velocities corresponding with large standard deviations.

Good agreement is obtained between the experimental data and the numerical simulations. In the laminar regimes, the numerical solution shows very little variation in the velocity field. This is expected, since the numerical inflow profile is strictly constant. In contrast, the small, but nearly constant experimental standard deviation obtained in these regimes is best explained as the signature of the background (undisturbed) atmospheric turbulence. It is also remarkably well-defined at around $5 \%$ of the reference velocity.

We also note that the numerical solution tends to somewhat over-predict the intensity of the turbulent fluctuations. This is also consistent with the tendency to under-predict the average velocities in turbulent regime, apparent in figure 10. We believe that this trend can be explained by the lack of any sub-grid turbulent viscosity in our model. If a turbulence model based on a sub-grid turbulent viscosity was introduced, increased momentum diffusion in the turbulent regime would lead to 

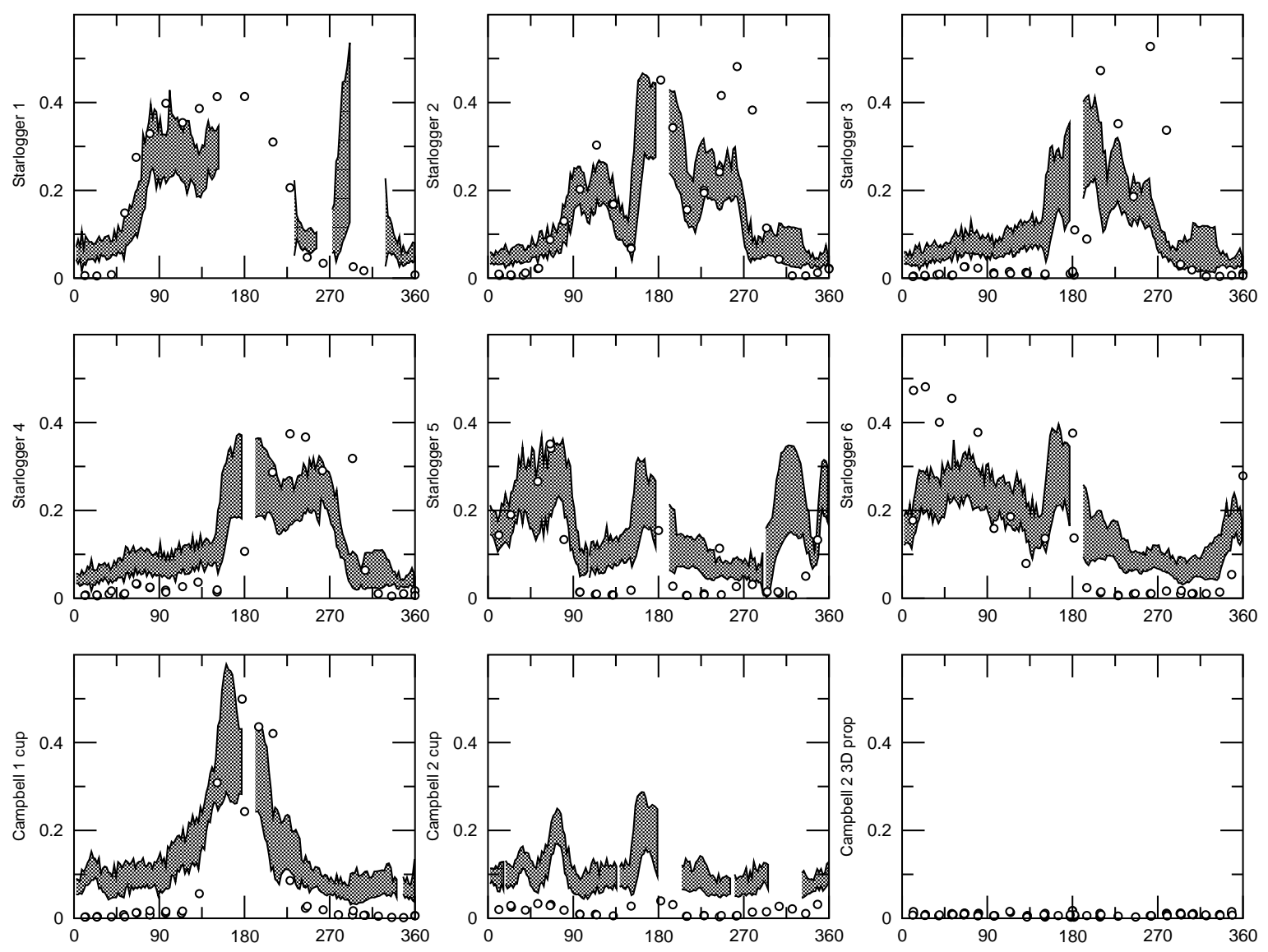

Figure 12: Normalised standard deviations of the wind speeds at each location as functions of relative wind direction. The experimental data is represented by the bounding curves defined by: mean plus or minus standard deviation. The symbols are the results of numerical simulations. 


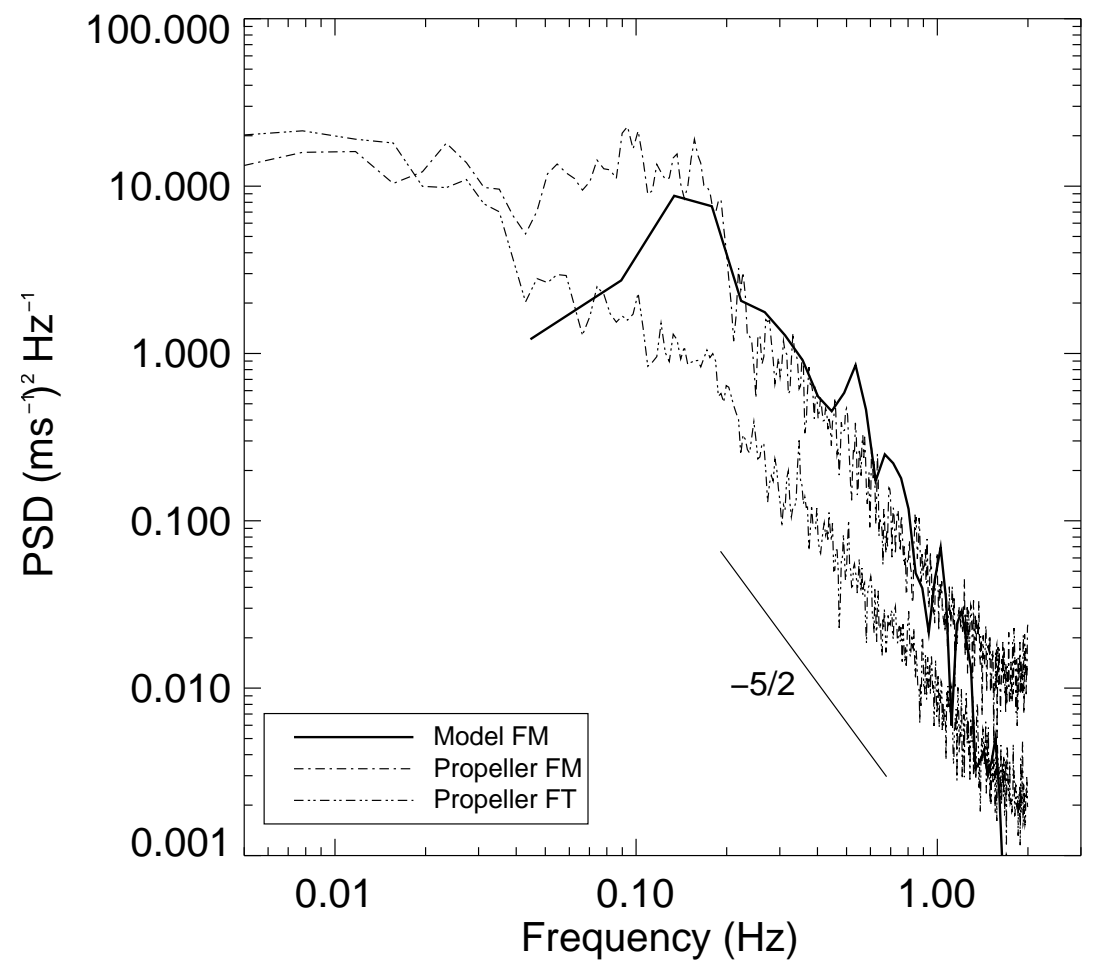

Figure 13: Comparison of modelled downwind power spectral density of velocity (solid line) with measured spectra upwind (dotted) and downwind (dot-dash). The relative wind speed was $10 \mathrm{~m} / \mathrm{s}$ from astern.

smaller velocity fluctuations and larger average velocities.

Another interesting feature of figure 12, when examined together with figure 10 is the correlation of increased turbulence with the small features seen for the Campbell 1 cup and Campbell 2 cup sites (near 15 and 75, 180 degrees respectively). This tends to confirm our hypothesis that these local average velocity variations are caused by small-scale upstream obstructions. The signature of a similar upstream obstruction, though of larger spatial extend, is clearly seen near 180 degrees for the Starlogger 6 location and is reproduced by the numerical simulation. It corresponds to one of the legs of the fantail moving upstream of the instrument location. This is clearly illustrated by the complex shape of the streamline going through this location for a stern-on wind (figure 9).

\section{d. Turbulence spectra}

The standard deviation of the velocity is only an integrated measure of the turbulent energy content of the signal measured. Being time-dependent, LES simulations give information about the detailed spatial distribution of turbulent structures down to scales comparable with the grid size. Figure 13 gives spectra obtained from experimental and numerical time series. The experimental spectra were calculated from segments of 1024 points averaged over 30 minutes. Two experimental locations are used: the foremast 3D propeller (Campbell $13 \mathrm{D}$ prop) and the fantail propeller (Campbell 2 $3 \mathrm{D}$ prop). Controlled comparisons between a sonic anemometer and the propellers showed that the response of the propellers starts to roll off above $0.4 \mathrm{~Hz}$, in keeping with early studies of these instruments (Horst 1973). For this comparison we deliberately chose the difficult situation of turbulent airflow in the wake of the vessel with the wind almost directly astern (165 degrees).

It is clear that the total energy content of the measured downwind turbulent spectrum exceeds the upwind spectrum by at least a factor of 6 , indicating that the turbulent wake intensity significantly exceeds the background atmospheric turbulence intensity (which is consistent with the standard deviation data of figure 12/Campbell 1 cup). The modelled wake spectrum agrees very well with the 


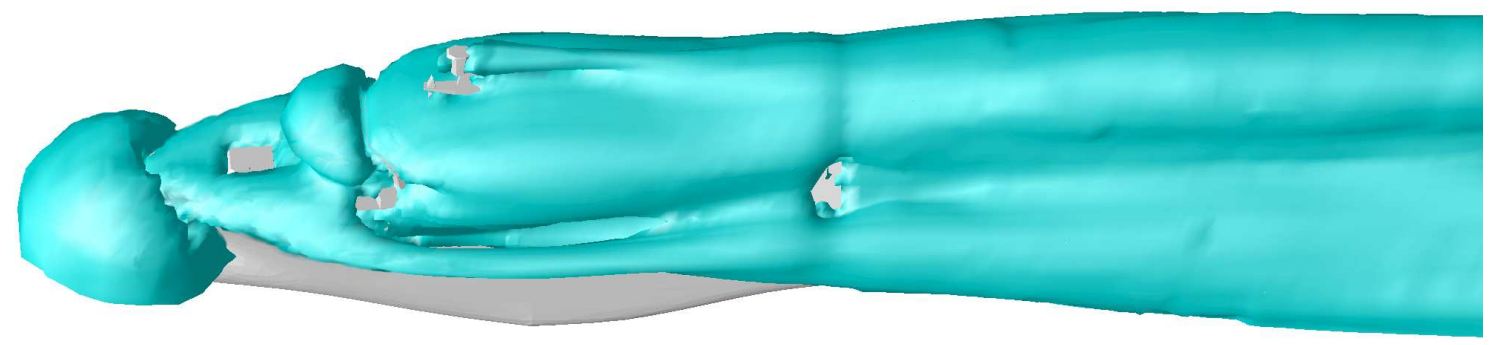

Figure 14: Isosurface at $90 \%$ of the time-averaged wind velocity for a bow-on flow. The velocity inside the volume pictured is lower than $90 \%$ of the inflow velocity.

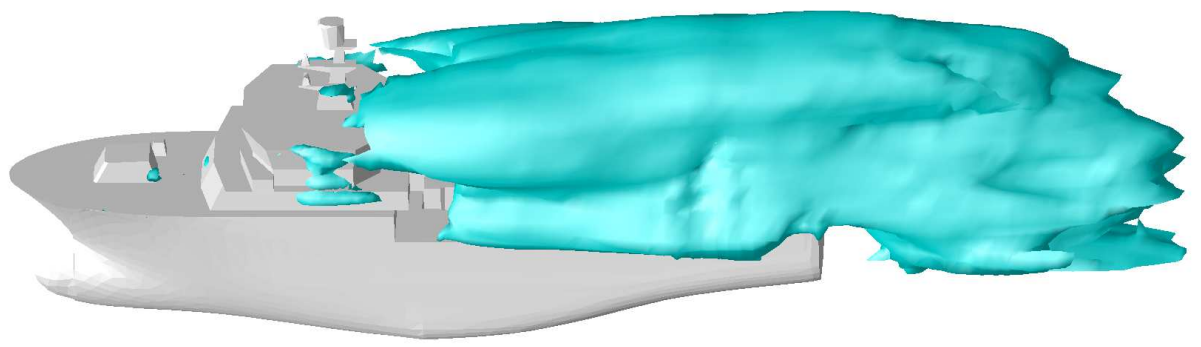

Figure 15: Isosurface at $25 \%$ of the normalised standard deviation for a bow-on flow. The standard deviation of the velocity inside the volume pictured is larger than $25 \%$ of the inflow velocity.

measured spectrum both qualitatively and quantitatively. The modelled spectrum does show a faster falloff above $1 \mathrm{~Hz}$ which we attribute to the finite spatial resolution of the model. Further model runs at higher resolution (not shown) extended the cutoff region to higher frequencies as expected (??). The modelled spectrum peaks at $0.15 \mathrm{~Hz}$, corresponding to a dominant eddy scale size of $\approx 66 \mathrm{~m}$ which is close to the scale size of the ship length. The modelled fall-off at lower frequencies indicates that no spatial structures larger than the ship length are created. This is consistent with vortex shedding occurring at scales comparable to the ship length and then decaying into smaller structures. At low frequencies (below $0.05 \mathrm{~Hz}$ ) the upwind and downwind experimental spectra converge indicating that at the corresponding length scales the influence of the ship on the background atmospheric turbulence is negligible. In the 0.04-0.2 Hz frequency range, ship motion (more particularly changes in relative wind direction due to yaw) typically have a strong influence on the measured spectrum.

\section{e. General characteristics}

One of the strength of the numerical simulations is that they give a global picture of the flow structure which is difficult to infer from point measurements. Three-dimensional maps characterising various measures of flow distortion are easily obtained. As an example, figure 14 uses an isosurface at $90 \%$ of the time-averaged velocity to illustrate the 3D structure of the velocity field. The large pressure building up at the bow and in front of the central superstructure creates the two rounded low-velocity zones in these areas. These two features would be described by a laminar potential flow approximation. Most of the other features are linked to vorticity generation at the ship boundary and subsequent advection by the flow. Particularly noticeable features are: the wake of the whole ship extending far into the domain, the wake created by the crow's nest and a tubular structure starting near the bow and extending the whole length of the ship. Closer examination reveals that this low velocity zone corresponds to the core of a longitudinal vortex fed by the strong vorticity generation near the bow. 


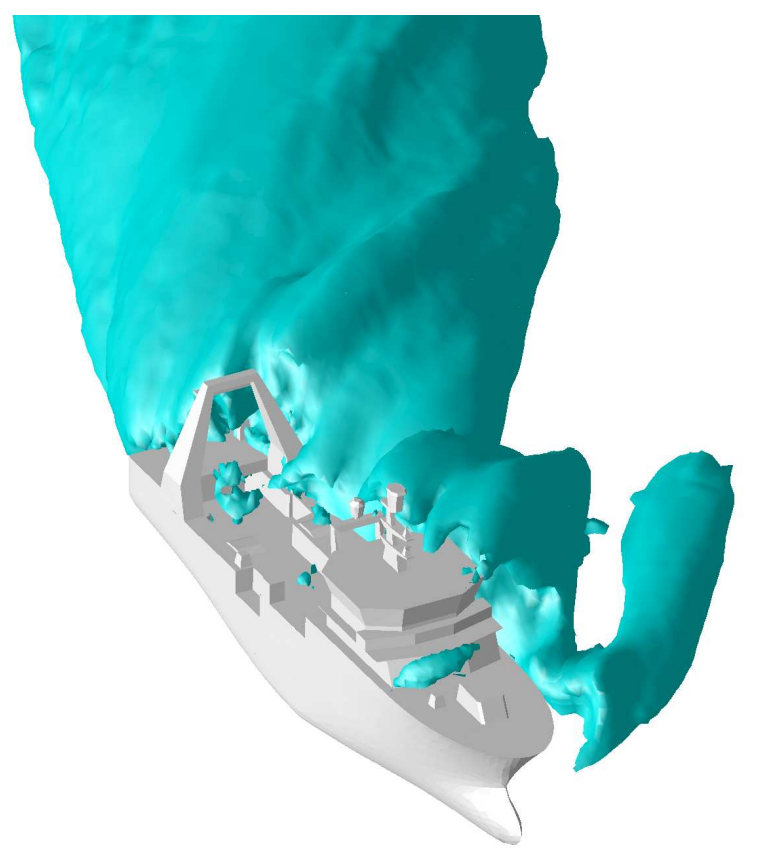

Figure 16: Isosurface at $25 \%$ of the normalised standard deviation for a 45 degrees wind flow. The standard deviation of the velocity inside the volume pictured is larger than $25 \%$ of the inflow velocity.

Using only figure 14, it is difficult to gauge of the velocity fluctuations, although one might guess that the downstream wake is turbulent while the upstream part of the flow is more or less stationary. Figure 15 uses the same type of representation but for the normalised standard deviation. A clear qualitative and quantitative picture of the strongly turbulent wake just downstream of the ship is obtained. It is interesting to note that, while the wake extends very far from the ship as seen on figure 14, the fluctuations tend to decrease rapidly when the distance to the ship increases. It is also seen that the bow vortices described in figure 14 are not associated with any significant fluctuation in velocity (i.e. they are stationary).

Figure 16 is a similar representation but for a 45 degrees relative wind direction. A much wider turbulent wake is generated with several clearly defined sub-wakes linked to specific parts of the ship. Particularly interesting is the strongly turbulent bow wake.

\section{f. Application to micro-meteorological measurements}

Figure 17.(a) and 17.(b) illustrate a characterisation of flow distortion at location "Campbell 1 3D prop" which is the main location used for micro-meteorological measurements on the Tangaroa. Both the full LES solution and the initial potential flow solution are given. Figure 17.(a) gives the relative vertical displacement of a parcel of air reaching the measurement location as a function of relative wind direction. This value is computed from the numerical results by following the time-averaged streamline passing through the instrument location. The vertical displacement of 1.5 meters for a bow-on flow and 6 meters for a 90 degrees relative wind direction are comparable to results obtained by (Yelland et al. 1998, 2002). Figure 17.(b) illustrates the dependence in relative wind direction of the wind speed measured relative to the inflow (exact) wind speed. The obstruction by the ship for a bow-on flow causes an under-estimation of $7 \%$ of the wind speed, while for a 90 degrees relative wind direction the wind speed is over-estimated by $10 \%$. These corrections will be applied to the determination of $C_{D}$ in future work. It is interesting to note that, while the potential flow solution gives a reasonable prediction of the relative wind speed for bow-on flows, it severely under-predicts the elevation for all relative wind directions.

The experiments carried out on the Tangaroa have validated the ability of the Gerris CFD model to 


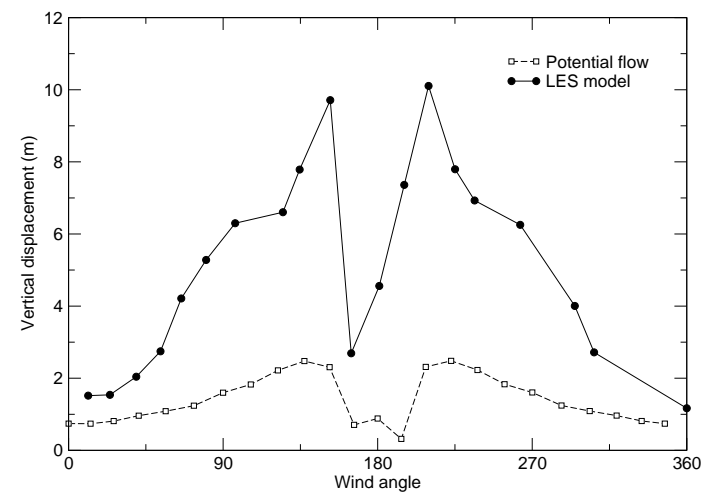

(a)

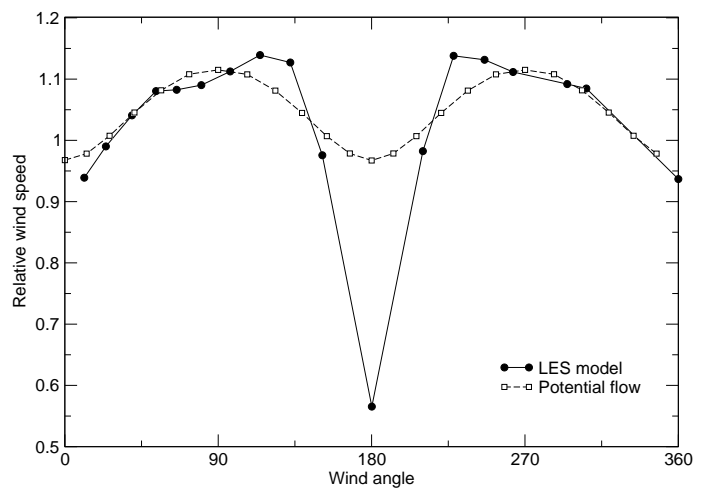

(b)

Figure 17: Characterisation of flow distortion at location "Campbell 1 3D prop". (a) Vertical displacement. (b) Relative wind speed.

simulate both time-averaged flow and time-varying turbulent structure. We are now able to consider specific problems relating to both the generation and distortion of turbulence by flow disturbance. In particular the region in front of the bow has been used as a gas flux profiling site in several recent experiments (e.g. (McGillis et al. 2001)), yet it is subject to effects due to pressure build up as shown in figure 14. We are now confident that the CFD model can be used to examine the effect of the ship on turbulent transfer at this location. This will be the subject of further study.

\section{Conclusion}

The experimental data set collected as part of this study confirms that the mean flow characteristics are only weakly dependent on ship motion, ship speed, wind speed or sea state, but strongly dependent on the relative wind direction. A new finding is that the normalised wind speed standard deviation (square-root of the turbulent kinetic energy) is also well characterised as a function of relative wind direction only. The standard deviation of the background atmospheric flow measured by well-exposed instruments is consistently close to $5 \%$ of the incoming wind speed. For badly exposed instruments, located in the wake of the ship superstructure, normalised standard deviations as high as $40 \%$ can be observed. The experimental data also confirm that even quite small structural elements (such as instruments mountings) can cause significant flow distortion.

Numerical studies performed using our time-dependent LES code show a very good agreement with both experimental mean velocities and standard deviations. These results have been obtained for the whole range of relative wind directions (from bow-on to stern-on) and remain valid in zones of high turbulence and high flow distortion. We also made use of the time-dependent nature of LES to obtain turbulence spectra. They are in excellent agreement with experimental data. The adaptive mesh technique we use has thus proved to give fast and accurate solutions for turbulent flows. These solutions are particularly useful when a global understanding of the flow pattern is sought, in order for example to optimise sampling location. The results also provide correction factors which can be applied to calculations of drag coefficients.

This work provides a validated basis for future studies. Although promising, the spectral analysis presented here is only preliminary and relies on a limited experimental dataset due to technical constraints (low sampling rates and high response time of most of the anemometers used). In the future we intend to carry out a more extensive measurement campaign using sonic anemometers. From a numerical modelling perspective, the simulation of turbulent flows around bluff bodies is still very much a work in progress in need of improvements (Shah and Ferziger 1997; Rodi et al. 1997; Iacacarino et al. 2003). Finally, by providing an open source version of the code which can be freely redistributed and modified (Popinet 2002), we hope to encourage research and collaboration in this field. 


\section{References}

Almgren, A. S., J. B. Bell, P. Colella, and T. Marthaler, 1997: A Cartesian grid projection method for the incompressible Euler equations in complex geometries. SIAM J. Sci. Comp., 18.

Almgren, A. S., J. B. Bell, and W. Y. Crutchfield, 2000: Approximate projection methods: Part I. Inviscid analysis. SIAM Journal on Scientific Computing, 22, 1139-1159.

Baetke, F., H. Werner, and H. Wengle, 1990: Numerical simulation of turbulent flow over surfacemounted obstacles with sharp edges and corners. J. Wind Eng. Ind. Aero., 35, 129-147.

Bell, J. B., P. Colella, and H. M. Glaz, 1989: A second-order projection method for the incompressible Navier-Stokes equations. J. Comput. Phys., 85, 257-283.

Boris, J. P., F. F. Grinstein, E. S. Oran, and R. L. Kolbe, 1992: New insights into large-eddy simulation. Fluid Dyn. Res., 199-228.

Brown, D. L., R. Cortez, and M. L. Minion, 2001: Accurate projection methods for the incompressible Navier-Stokes equations. J. Comput. Phys., 168, 464-499.

Brut, A., A. Butet, S. Planton, P. Durand, and G. Caniaux: 2002, Influence of the airflow distortion on air-sea flux measurements aboard research vessel: results of physical simulations applied to the Equalant99 experiment. 15th AMS Conf. Boundary Layer \& Turbulence, Wageningen, Netherlands.

Chorin, A. J., 1968: Numerical solution of the Navier-Stokes equations. Math. Comp., 22, 745-762.

Dupuis, H., C. Guerin, D. Hauser, A. Weill, P. Nacass, W. Drennan, S. Cloch, and H. Graber, 2003: Impact of flow distortion corrections on turbulent fluxes estimated by the inertial dissipation method during the FETCH experiment on R/V L'Atalante. J. Geophys. Res., C3.

Horst, T. W., 1973: Corrections for response errors in a three-component propeller anemometer. $J$. Appl. Meteorol., 12, 716-725.

Iacacarino, G., A. Ooi, P. A. Durbin, and M. Behnia, 2003: Reynolds averaged simulation of unsteady separated flow. Int. J. Heat and Fluid Flow, 24, 147-156.

Kahma, K. K. and M. Lepparanta, 1981: On errors in wind speed observations on R/V Aranda. Geophysica, 17, 155-165.

Khokhlov, A. M., 1998: Fully threaded tree algorithms for adaptive refinement fluid dynamics simulations. J. Comput. Phys., 143, 519-543.

Lesieur, M. and O. Métais, 1996: New trends in large-eddy simulations of turbulence. Ann. Rev. Fluid Mech., 28, 45-82.

McGillis, W. R., J. B. Edson, J. D. Ware, J. W. H. Dacey, J. E. Hare, C. W. Fairall, and R. Wanninkhof, 2001: Carbon dioxide flux techniques performed during GasEx-98. Marine Chemistry, 75, 267-280.

Murakami, S., 1993: Comparison of various turbulence models applied to a bluff body. J. Wind Eng. Ind. Aero., 46, 21-36.

Oost, W. A., C. Fairall, J. Edson, S. Smith, R. Anderson, J. Wills, K. Katsaros, and J. DeCosmo, 1994: Flow distortion calculations and their application in HEXMAX. J. Atmos. Oceanic Technol., 11, 366386.

Pedreros, R., G. Dardier, H. Dupuis, H. Graber, W. Drennan, A. Weill, C. Guérin, and P. Nacass: 2003, Momentum and heat fluxes via the eddy correlation method on the R/V L'Atalante and an Asis buoy, submitted to J. Geophys. Res.

Peyret, R. and T. D. Taylor, 1983: Computational Methods for Fluid Flow. Springer Verlag, New York/Berlin. 
Popinet, S., 2002: The Gerris Flow Solver. http://gfs.sourceforge.net.

- 2003: Gerris: a tree-based adaptive solver for the incompressible Euler equations in complex geometries. J. Comp. Phys., 190, 572-600.

Porter, D. H., A. Pouquet, and P. R. Woodward, 1994: Kolmogorov-like spectra in decaying threedimensional supersonic flows. Phys. Fluids, 6, 2133-2142.

Quirk, J. J., 1994: An alternative to unstructured grids for computing gas dynamics flows around arbitrarily complex two-dimensional bodies. Computers and Fluids, 23, 125-142.

Rodi, W., J. H. Ferziger, M. Breuer, and M. Pourquie, 1997: Current status of large-eddy simulations: results of a workshop. ASME J. Fluids Eng., 119, 248-262.

Samet, H., 1989: Applications of Spatial Data Structures. Addison-Wesley Publishing Company.

Shah, K. B. and J. H. Ferziger, 1997: A fluid mechanician's view of wind engineering: large-eddy simulation of flow past a cubical obstacle. J. Wind Eng. Ind. Aero., 67/68, 211-224.

Thiebaux, M. L., 1990: Wind tunnel experiments to determine correction functions for shipborne anemometers. Canadian Contractor Report of Hydrography and Ocean Sciences 36. Technical report, Beford Institute of Oceanography, Dartmouth, NS, Canada.

Ye, T., R. Mittal, H. S. Udaykumar, and W. Shyy, 1999: An accurate cartesian grid method for viscous incompressible flows with complex immersed boundaries. J. Comp. Phys., 156, 209-240.

Yelland, M., B. Moat, R. Pascal, and D. Berry, 2002: CFD model estimates of the airflow distortion over research ships and the impact on momentum flux measurements. J. Atmos. Oceanic Technol., 19, 1477-1499.

Yelland, M., B. Moat, P. Taylor, R. Pascal, J. Hutchings, and V. Cornell, 1998: Wind stress measurements from the open ocean corrected for airflow distortion by the ship. J. Phys. Oceanogr., 28, $1511-1526$. 